\title{
Study of Clinical And Laboratory Profile of Sjogren's Syndrome.
}

\author{
Lalana Kalekar*, Namita Padwal, **, Sijju Pillai*** \\ *Associate Professor, Department Of Medicine, LTMGH, Sion, Mumbai. \\ **Associate Professor, Department Of Medicine, LTMGH, Sion, Mumbai \\ ***Senior Resident, Department Of Medicine, LTMGH, Sion, Mumbai.
}

\begin{abstract}
:
Objective: To study clinical and laboratory profile of Sjogren's syndrome.

Method and Material: Total of 27 patients of Sjogren's syndrome (15 Primary \& 12 Secondary) were recruited in the study. As per American-European Consensus criteria, Sjogren's syndrome was diagnosed, when patient satisfied 4 of the six criteria.

Results: Total 27 patients were studied, 15 were diagnosed as Primary Sjogren's syndrome and 12 were diagnosed as Secondary Sjogren's syndrome, as per the criteria. Out of 15 patients, who had Primary Sjogren's syndrome, 12 were female patients and 3 were male patients. 3 males and 9 females were studied, who had Secondary Sjogren's syndrome, (M: F=2:8). Mean age of diagnosis was 44 years in Primary Sjogren's syndrome.
\end{abstract}

Conclusions: Sjogeren's syndrome is common. Xerostomia and xeropthalmia are common symptoms. Along with serology, Lip salivary gland biopsy can confirm diagnosis.

\section{Introduction}

Sjogren's syndrome (SS) is a chronic \& slowly progressive autoimmune disease, characterized by lymphocytic infiltration of the exocrine glands resulting in xerostomia \& exophthalmia. This leads to progressive destruction of the glands resulting in, dry eye \& dry mouth, the two cardinal symptoms of the disease ${ }^{1}$.

Sjogren's syndrome, was first diagnosed by, Swedish doctor, Henrick Sjogren in $1933^{2}$ previously, in 1882, Leber had described filamentary hepatitis, and in 1888, Mikulicz described a patient with bilateral lacrimal and parotid enlargement. Biopsy of these glands revealed extensive round-cell infiltration. In 1953, Morgan and Castleman noted the commonality between the glandular enlargement described by Mikulicz and the keratitis described by Sjögren. Subsequently, these disorders were considered to be variants of the same process and the term Sjögren's syndrome (SS) became more widely used ${ }^{3}$.

Extra - glandular involvement of muscle, blood vessel, lung and kidney also may be seen. It may exist alone (Primary Sjogren's syndrome) or occur in association with other connective tissue diseases (Secondary Sjogren's syndrome). The disease spectrum extends from an organ specific autoimmune disease (autoimmune exocrinopathy) to a systemic process (including musculoskeletal, pulmonary, gastric, hematological, renal \& nervous system involvement). Antibodies to SSA (Ro) and SSB (La) are characteristically associated with it. ${ }^{4}$

SS primarily affects females, during fourth and fifth decade of life. The female to male ratio is 9:1. One of the most striking characteristics of primary Sjogren's syndrome is its higher prevalence among, middle aged woman with a Female: Male ratio of 9:1. Sjogren's syndrome, although common disorder in Western countries with an estimated prevalence of 3 in 100 to 1 in $100{ }^{5}$, has rarely been reported from India ${ }^{6,7}$. Lack of awareness of this entity, by attending physicians, ophthalmologists \& dentists may be one of the factors.

The main Sicca symptoms (xerophthalmia \& xerostomia) are detected by specific ocular tests (Rose Bengal Staining, Schirmer's test) \& oral tests (Salivary flow measurement, parotid scintigraphy).

The histological hallmark is a focal lymphocytic infiltration of the exocrine glands, determined by a biopsy of, the minor salivary glands.

Patients with Sjogren's syndrome presents, a broad spectrum of, serological features (cytopenia, hypergammaglobulinemia, high ESR) and auto antibodies of which ANA are most frequently detected, Anti SSB antibody the most specific .

The question that arises is, whether the clinical \& immunological manifestations of primary Sjogren's syndrome in men, differs from, those in women. This is important because the examination of more homogenous subset, such as male patients, may allow for earlier diagnosis with better prognosis.

As Sjogren's syndrome, is rarely reported, from our country, we decided to study, the clinical \& immunological features, of our patients. We also analyzed the clinical manifestation \& immunological features of males \& females with, primary Sjogrens syndrome, to determine whether any relation exists between, disease 
pattern \& gender. We also analyzed, age at diagnosis $(\leq 35 \mathrm{yrs} \&>36 \mathrm{yrs})$ as an independent variable, to determine whether any relation exists between disease pattern $\&$ age of diagnosis.

\section{Materials and methods}

This is an observational, single centre, prospective study carried out at a tertiary care hospital over a period of 18 months in patients with Sjogren's syndrome.

All patients attending Rheumatology services at tertiary care hospital, indoor \& outdoor, fulfilling the European - American Consensus Group Modification of the European Community Criteria for Sjogren's syndrome ${ }^{8}$ were included in the study. The study protocol was approved by Ethics \& Research committee.

Patients- The study included 27, consecutively diagnosed patients with Sjogren's syndrome, seen at the "Rheumatology OPD" of this hospital, over a period of, 18 months. Diagnosis was made, according to European-American consensus group modification of the European community criteria.

\section{European-American Consensus Group Modification Of The European Community Criteria For Sjögren's Syndrome (Ss) ${ }^{8 .}$}

\begin{tabular}{|l|}
\hline I. Symptoms of dry eye \\
\hline 1.Patients must have a positive response to at least one of the following: \\
\hline 2. Have you had daily, persistent, troublesome dry eyes for more than 3 months? \\
\hline 3. Do you have a recurrent sensation of sand or gravel in the eyes? \\
\hline 4. Do you use tear substitutes more than 3 times a day? \\
\hline II. Oral symptoms \\
\hline 1.Patients must have a positive response to at least one of the following: \\
\hline 2. Have you had a daily feeling of dry mouth for more than 3 months? \\
\hline 3. Have you had recurrently or persistently swollen salivary glands as an adult? \\
\hline 4. Do you frequently drink liquids to aid in swallowing dry food? \\
\hline III. Ocular signs \\
\hline Patients must have objective evidence of ocular involvement, defined as a positive result from at least one of the following \\
two tests: \\
\hline 1) Schirmer's test performed without anesthesia (5 mm in 5 min). \\
\hline 2) Rose Bengal score or other ocular dye score (4 according to van Bijsterveld's scoring system) \\
\hline
\end{tabular}

\section{Histopathology}

This criterion is met if in patient's minor salivary glands (obtained through normal-appearing mucosa) focal lymphocytic sialadenitis is evaluated by an expert histopathology's with a focus score of 1 . This score is defined as the number of lymphocytic foci adjacent to normalappearing mucous acini and containing more than 50 lymphocytes per $4 \mathrm{~mm}^{2}$ of glandular tissue.

\section{Salivary gland involvement} diagnostic tests:

Patient must have objective evidence of salivary gland involvement defined by a positive result for at least one of the following

1) Unstipulated whole salivary flow $(1.5 \mathrm{ml}$ in $15 \mathrm{~min})$

2) Parotid sialography showing the presence of diffuse sialectasis, punctuate, cavitary, or destructive pattern) without evidence of obstruction in the major ducts

3) Salivary scintigraphy showing delayed uptake, reduced concentration, delayed excretion of tracer, or some combination of these

\section{Autoantibodies}

Patient must have presence in the serum of the following autoantibodies:

Antibodies to Ro(SS-A) or La (SS-B)

Primary Sjögren's syndrome: any four of the six items, as long as item IV (histopathology) or VI (serology) is positive, or any three of the four objective criteria items (items III, IV, V, and VI). In patients with a potentially associated disease (e.g., another well-defined connective tissue disease), the presence of item I or item II plus any two from among items III, IV, and V may be considered as indicative of secondary Sjögren's syndrome. Patients with past history of head and neck irradiation treatment, Hepatitis $\mathrm{C}$ infection, AIDS, pre- 
existing lymphoma, Sarcoidosis, GVHD, use of anti-cholinergic were excluded. An investigator administered questionnaire was used to gather data prospectively.

\section{Study Design}

All patients attending, Rheumatology services, indoor \& outdoor, were questioned regarding the presence of dry eye \& / or dry mouth .If present, Schirmer's test \& / or Rose Bengal staining was carried out to confirm xerostomia. Schirmer's test was considered positive, when the wetting of $30 \mathrm{~mm}$ filter paper inserted in the lower eyelid, was $<5 \mathrm{~mm}$ in 5 minutes. Rose Bengal staining was used to identify devitalized epithelium of the cornea \& conjunctiva as punctuate or filamentary keratitis under slit lamp. Abnormal unstimulated, whole salivary flow (1.5 $\mathrm{ml}$ in 15 minutes), with or without presence of caries teeth was considered as xerostomia .Associated autoimmune diseases like Rheumatoid Arthritis ,SLE, Systemic sclerosis \& Inflammatory myositis were screened.

Routine laboratory investigations included complete blood count, erythrocyte sedimentation rate ( ESR ), blood urea nitrogen (BUN ), serum creatinine, serum electrolytes, total proteins \& globulin levels, urine analysis, chest X-ray, arterial blood gas analysis. Anti SSA (Ro) \& Anti SSB (La) antibody testing was carried out by commercially available kits. Nerve conduction studies were used to establish diagnosis of neuropathy. ANA, RA factor \& serum protein electrophoresis were obtained for all patients.

Patients of sicca syndrome (dry eyes and dry mouth) underwent minor salivary gland biopsy \& autoantibody screen. Biopsy of the minor salivary gland from the lower lip was carried out, to assess for lymphocytic infiltration. A focus score defined $>2$ of the 4 evaluable lobes was considered as evidence of involvement. A focus is defined as $>50$ lymphocytes per $\mathrm{mm}^{2}$.

Data regarding extra glandular features like arthritis, renal, gastrointestinal, neurological, pulmonary, vascular \& cutaneous involvement were retrieved from patients.

\section{Limitation Of Study}

Present study was carried out at a single centre, within established cohort of small number of patients. Thus selection bias may contribute to the percentages of various manifestations observed.

\section{Results}

In our study, out of total 27 patients, 15 patients with primary Sjogren's syndrome, out of which, 12 $(80 \%)$ were females and $3(20 \%)$ were males. Male: Female ratio was 2:8, with mean age at diagnosis of 44.27 years (range $18-64$ year).

The presenting symptoms were xerostomia and xerophthalmia in 11 patients $(73.3 \%)$, articular involvement in $10(66.6 \%)$, hypokalemic paralysis in $1(6.6 \%)$ and jaundice in $1(6.6 \%)$. ANA was positive in $8(53.3 \%)$ patients, Rheumatoid factor in $8(53.3 \%)$, SS-A (Ro) in 9(60\%); SS-B (La) in 9(60\%).

Of the extra glandular manifestations: articular involvement in $10(66.6 \%)$, Reynaud's phenomenon in $1(6.6 \%)$, vasculitis in $2(13.3 \%)$, peripheral neuropathy in $4(26.6 \%)$, distal renal tubular acidosis in $3(20 \%)$, purpura in $2(13.3 \%)$, autoimmune hepatitis in $1(6.6 \%)$, autoimmune thyroiditis in $1(6.6 \%)$, hypergammaglobulinemia in $12(80 \%)$, fatigue was the most common symptom seen in $12(80 \%)$, headache was seen in $10(66.6 \%)$.

In 6 patients, primary Sjogren's syndrome was diagnosed before the age of 40 years, of which 5 were female and 1 male. A higher prevalence of immunological markers i.e. RA(83.3\%), Anti SS-A(83.3\%), Anti $\mathrm{SS}-\mathrm{B}(83.3 \%)$ were found in patients $\leq 40$ years of age as compared with $33.3 \%, 44.4 \% \& 44.4 \%$ respectively among those aged $>40$ years, but it did not reach statistical significance. There was no statistically significant difference in the occurrence of other manifestations.

Of the 15 patients, 12 were females and 3 males. Females $(75 \%)$ had a higher prevalence of articular involvement when compared to males $(33.3 \%)$ but it did not reach statistical significance. There was no other statistically significant difference in the occurrence of xerostomia, xerophthalmia, and peripheral neuropathy. Although the prevalence of immunological markers i.e. Anti - Ro (SS-A), Anti - La(SS-B) \& Rheumatoid factor were slightly higher in females as compared to males, it did not reach statistical significance . Fatigue was seen in $100 \%$ of cases among females which was significantly higher as compared to not a single patient among male cases. Lip salivary gland biopsy was done in 11 patients who had sicca syndrome.

Of the 12 patients with secondary Sjogren's syndrome $9(75 \%)$ were females \& 3(25\%) were males. Xerostomia was seen in $10(83.3 \%)$, xerophthalmia in 10(83.3\%), articular involvement in $10(83.3 \%)$, peripheral neuropathy in $3(25 \%)$, renal tubular acidosis in $2(16.6 \%)$, parotid enlargement in $1(8.3 \%)$, purpura in $3(25 \%)$. Among the immunological markers RF in $5(41.6 \%)$, ANA in $4(32.5 \%)$, Anti SSA antibody in $5(41 \%)$, Anti SSB antibody in 5(41\%), hypergammaglobulinemia in 7(58.3\%). Of the 12 patients $5(41 \%)$ had Rheumatoid Arthritis, 4(33.3\%) had SLE \& 3(25\%) had Scleroderma. 


\section{Limitation Of Study}

Present study was carried out at a single centre, within established cohort of small number of patients. Thus selection bias may contribute to the percentages of various manifestations observed.

\section{Discussion}

Sjogren's syndrome has been infrequently reported in Indian subcontinent. A population based study of rheumatic disease in India ${ }^{9}$, conducted by a rheumatology centre, makes no mention of this disease; whereas in Western countries, the prevalence varies depending upon the criteria used, thus using the European Economic Criteria in Europe, the prevalence is 1-3 \% , whereas using the San Diego or San Francisco criteria the figure is $0.5 \%$ in USA.

The controversy surrounding the diagnostic criteria of Sjogren's syndrome persists \& there are as many as 7 different criteria with varying specificities \& sensitivities .In present study, we have used the European American Consensus Group modification of the European Community Criteria for Sjogren's syndrome ${ }^{8}$.

Primary Sjogren's syndrome is an autoimmune disease primarily affecting females in the fourth \& fifth decade of life as seen in our study also. Table no. 1 indicates that of the 12 females with primary Sjogren's syndrome 5(41\%) were of the age group 51-60. Of the 3 males 2(66.6\%) were of the age group 51-60. In our study the youngest patient was 18 years old. In comparison the youngest patient reported in literature was 3 years old ${ }^{10}$.

In present study, the mean age of diagnosis of our patients with primary Sjogren's syndrome is 10 years younger, than that reported from, European studies by Ramos-Casals et al ${ }^{11}$, Atamans et al ${ }^{12}$, Theander et $\mathrm{al}^{13}$ and Garcia - Carrasco et al ${ }^{14 .}$ This "shift to the left" phenomenon is probably due to less proportion of geriatric patients in our population. (Table2). In our study, the Male: Female ratio was 2:8 which was similar to observations made by previous studies on Indian population by R.Mishra ${ }^{15}$ et al and R.Porkodi ${ }^{16}$ et al .Our gender bias for females is less as compared to European studies by Ramos-Casals et al ${ }^{11}$, Alamanos et al ${ }^{12}$, Theander et al ${ }^{13}$, Ioannidis et al ${ }^{17}$ and Garcia - Carrasco et al ${ }^{14}$.

The controversy surrounding the diagnostic criteria of Sjogren's syndrome persists \& there are as many as 7 different criteria with varying specificities \& sensitivities .In present study, we have used the European American Consensus Group modification of the European Community Criteria for Sjogren's syndrome ${ }^{8}$.

(Table 3)There is reservation of getting a biopsy by patients and physicians and many Rheumatologists feel that documentation of dry eye $\&$ dry mouth along with presence of antibodies abrogates the need for salivary gland biopsy. In our country, where autoantibody testing is still not widely available, biopsy is a simple \& definitive test for diagnosis if adequate tissue is collected at biopsy. It also helps in excluding diagnosis like tuberculosis, sarcoidosis \& lymphoma, which mimic the clinical picture of Sjogren's syndrome. (Table 4).

Females with primary Sjogren's syndrome had higher prevalence of articular Involvement \& immunological markers i.e. Anti SSA (Ro), Anti SSB (La) \& RF as compared to males. (Table 5).

\section{Conclusions}

Primary Sjogren's syndrome is, an autoimmune disease primarily affecting females in the fourth \& fifth decade of life, with Female: Male ratio of 8:2. Xerostomia and xerophthalmia were the most common presenting symptoms. Articular involvement was the most common extraglandular manifestation. ANA and RF was the most frequently detected antibody in primary Sjogren's syndrome.

Secondary Sjogren's was seen most commonly in association with Rheumatoid Arthritis. Xerostomia, xerophthalmia and auricular involvement were the most common manifestations.

Females with primary Sjogren's syndrome had higher prevalence of articular involvement \& immunological markers i.e. Anti SSA (Ro), Anti SSB (La) \& RF as compared to males, but it was not statistically significant. Fatigue among females was significantly higher as compared to males. No other significant difference in clinical and immunological characteristics of male and female patients with primary Sjogren's syndrome was found.

Prevalence of immunological markers i.e. Anti SSA (Ro), Anti SSB (La) \& RF was higher in patient's $\leq 40$ years of age, but it did not reach statistical significance. There was no significant difference in the occurrence of other manifestations.

Primary Sjogren's syndrome can present initially with protean manifestations to various specialists like the ophthalmologist, dentist, neurologist, nephrologists or gastroenterologist. The initial manifestation can be non specific and it may take more than 10 years, from the initial symptom to the full blown development of the syndrome.

\section{References}

[1]. Haralampos M. Moutspoulos Sjogren syndrome: Harrison's principles of Internal medicine $17^{\text {th }}$ edition, Vol 2: 2107-09.

[2]. Mason AM, Gumpel JM, Golding PL: Sjögren's syndrome: A clinical review. Sem Arthritis Rheum 1973; 2:301.

[3]. Tziooufas AG, Moutsopoulus HM. Sjögren's syndrome. In: Hochberg MC, Silman AJ, Smolen JS, Winblatt ME, Weisman MH, eds. Rheumatology, 3rd ed. London: Mosby 2003; 1431-1443. 
[4]. R Porkodi, S Rukmangathrajan, P Kanakarani, M Prathiban .Primary Sjogren syndrome - clinical and immunological features: Indian Rheumatol Assoc 2003:11:63-65.

[5]. Edward d Harris, Ralph Budd, and Shaun Ruddy: Kelley's textbook Rheumatology: $7^{\text {th }}$ Edition. AN, Venugopal B, Thenmozivilli PR. Spectrum of Clinical and immunological features of systemic rheumatic disorders in a referral hospital in south IndiaSjogrens syndrome s.J Indian Rheumatologic assoc.1994; 2:7

[6]. IMalavia AN, Kar K, Goswami S, Connective tissue disease in India clinical immunological studies in Sjogrens Syndrome. JAPI 1978; 26:553-9.

[7]. Vitali C, et al, Classification criteria for Sjogren's syndrome. A revised version of the European criteria proposed by the AmericanEuropean Consensus Group. Ann Rheum Dis 2002; 61:554-558.

[8]. Malavia AN, Singh RR, Kapoor SK. Prevalence of rheumatic diseases in India. Results of a population survey. J of rheumatic assoc 1994; 2:13-17.

[9]. Siamopoulou -Mauridou A, Drosos AA, Antonopoulos AP. Sjogren 's Syndrome in childhood: report of two cases. European Journal of Pediatrics 1989; 8:523-4.

[10]. Manuel Ramos-Casals, Roser Solans, Jose Rosas. Primary Sjogren's Syndrome in Spain Clinical and Immunologic Expression in 1010 Patient 2008; 87:210-219

[11]. Alamanos Y, Tsifetaki N, Voulgari PV, Venetsanopoulou AI, Siozos C, Drosos AA. syndrome in north-west Greece, 1982-2003. Rheumatology (Oxford). 2006; 45:187-191

Epidemiology of primary Sjogren's

[12]. Theander E, Manthorpe R, Jacobsson L. Mortality and causes of death in Primary Sjogren's syndrome: a prospective cohort study. Arthritis Rheum. 2004; 50:1262-1269.

[13]. Garcia-Carrasco M, Ramos-Casals M, Rosas J, Pallares L, Calvo-Alen J,Cervera R, Font J Ingelmo M. Primary Sjogren syndrome: clinical and Immunologic disease patterns in a cohort of 400 patients. Medicine (Baltimore). 2002; 81:270-280.

[14]. R.Mishra, P.Hissaria, Vini Tandon. Primary Sjogren's syndrome: Rarity in India. J AssocPhy India 2003; 51:859-62.

[15]. R.Porkodi, S.Rukumangatharajan, P.Kanakarani.Primary Sjogren's syndrome - Clinical and Immunological features.J Indian rheumatol assoc. 2003:11:63-65.

[16]. Ioannidis J, Vassiliou V, Moutsopoulos H. Long-term risk of mortality and lymphoproliferative disease and predictive classification of primary Sjogren's syndrome. Arthritis Rheum. 2002; 46:741-7.

Table no 1: Age and sex distribution of primary Sjogren's syndrome.

\begin{tabular}{|c|c|c|}
\hline Age group ( years ) & Male & Female \\
\hline $11-20$ & - & 1 \\
\hline $21-30$ & 1 & 1 \\
\hline $31-40$ & - & 3 \\
\hline $41-50$ & - & 5 \\
\hline $51-60$ & 2 & 1 \\
\hline
\end{tabular}

Table no 2: Comparison of our study of pt. with primary SS with International studies:

\begin{tabular}{|c|c|c|c|c|c|c|}
\hline & $\begin{array}{c}\text { Present } \\
\text { study }\end{array}$ & $\begin{array}{c}\text { Ramos-Casals } \\
\text { et al }{ }^{\mathbf{1 0}}\end{array}$ & $\begin{array}{c}\text { Ioannidis } \\
\text { et al }^{\mathbf{1 6}}\end{array}$ & $\begin{array}{c}\text { Alamanos et } \\
\mathbf{a l}^{\mathbf{1 1}}\end{array}$ & $\begin{array}{c}\text { Theander et } \\
\mathbf{a l}^{\mathbf{1 2}}\end{array}$ & $\begin{array}{c}\text { Garcia - Carrasco } \\
\text { et al }{ }^{\mathbf{1 3}}\end{array}$ \\
\hline $\begin{array}{c}\text { No of } \\
\text { patients }\end{array}$ & 15 & 1010 & 723 & 422 & 265 & 400 \\
\hline Country & India & Spain & Greece & Greece & Sweden & Spain \\
\hline Sex (F) & $80 \%$ & $94 \%$ & $94 \%$ & $95 \%$ & $91 \%$ & $93 \%$ \\
\hline F:M ratio & $8: 2$ & $13: 1$ & $16: 1$ & $20: 1$ & $10: 1$ & $14: 1$ \\
\hline $\begin{array}{c}\text { Xerostomi } \\
\text { a }\end{array}$ & $73 \%$ & $96 \%$ & $95 \%$ & $94 \%$ & & $98 \%$ \\
\hline $\begin{array}{c}\text { Xerophtha } \\
\text { lmia }\end{array}$ & $73 \%$ & $96 \%$ & $96 \%$ & $100 \%$ & & $93 \%$ \\
\hline
\end{tabular}

Table no3: Frequency of occurrence of diagnostic criteria of primary Sjogren's syndrome in the study

\begin{tabular}{|c|c|}
\hline \multicolumn{2}{|c|}{ population. } \\
\hline Criteria & No. (\%) \\
\hline Ocular tests & $13(86.6 \%)$ \\
\hline Xerophthalmia & $11(73.3 \%)$ \\
\hline Xerostomia & $(73.3 \%)$ \\
\hline Salivary gland abnormality & $11(73.3 \%)$ \\
\hline Minor salivary gland biopsy & $10(66.6 \%)$ \\
\hline Auto antibodies & $9(60 \%)$ \\
\hline
\end{tabular}

Table no. 4. Comparison of Clinical and lab profile of Primary and secondary SS.

\begin{tabular}{|l|l|l|l|}
\hline Sr. No. & Clinical \& Lab Profile & Primary Sjogrens Syndrome & Secondary Sjogrens Syndrome \\
\hline 1 & Total(n=27) & $\mathbf{1 5}$ & $\mathbf{1 2}$ \\
\hline 2 & Male $(\mathrm{n}=6)$ & $3(20 \%)$ & $3(25 \%)$ \\
\hline 3 & Female(n=21) & $12(80 \%)$ & $9(75 \%)$ \\
\hline 4 & Dry Mouth $(\mathrm{n}=18)$ & $11(73.3 \%)$ & $10(83.3 \%)$ \\
\hline 5 & Dry Eyes(n=18) & $11(73.3 \%)$ & $10(83.3 \%)$ \\
\hline 6 & Arthritis(n=20) & $10(66 \%)$ & $10(83.3 \%)$ \\
\hline 7 & Hypokalemic Paralysis $(\mathrm{n}=1)$ & $1(6.6 \%)$ & 0 \\
\hline 8 & Hepatitis(n=1) & $1(6.6 \%)$ & 0 \\
\hline
\end{tabular}


Study Of Clinical And Laboratory Profile Of Sjogren's Syndrome.

\begin{tabular}{|l|l|l|l|}
\hline 9 & Fatigue $(\mathrm{n}=27)$ & $15(100 \%)$ & $12(100 \%)$ \\
\hline 10 & Purpura(n=5) & $2(13.3 \%)$ & $3(25 \%)$ \\
\hline 11 & Hypergamaglobulinemia(n=19) & $12(12 \%)$ & $7(58 \%)$ \\
\hline 12. & Parotid swelling(n=1) & 0 & $1(8.33 \%)$ \\
\hline 13 & ANA(n=12) & $8(53.3 \%)$ & $4(35 \%)$ \\
\hline 14 & Anti DS DNA & 0 & $4(35 \%)$ \\
\hline 15 & RA Factor(n=13) & $8(53.3 \%)$ & $5(41.6 \%)$ \\
\hline 16 & Anti CCP & 0 & $5(41.6 \%)$ \\
\hline 17 & Anti Ro(n=14) & $9(60 \%)$ & $5(41.6 \%)$ \\
\hline 18 & Anti La(n=14) & $9(60 \%)$ & $5(41.6 \%)$ \\
\hline 19 & Reynaud's phenomenon(n=1) & $1(6.6 \%)$ & 0 \\
\hline 20 & Vasculitis(n=2) & $2(13.3 \%)$ & 0 \\
\hline 21 & Peripheral Neuropathy(n=7) & $4(26.6 \%)$ & $3(25 \%)$ \\
\hline 22 & Renal Tubular Acidosis(n=5) & $3(20 \%)$ & $2(16 \%)$ \\
\hline 23 & Rheumatoid Arthritis(n=5) & 0 & $5(41.6 \%)$ \\
\hline 24 & SLE(n=4) & 0 & $4(35 \%)$ \\
\hline 25 & Scleroderma(n=3) & 0 & $3(25 \%)$ \\
\hline 26 & Lip salivary gland biopsy & $11(73.3 \%)$ & $10(83.3 \%)$ \\
\hline
\end{tabular}

Table no.5: Age comparison of clinical \& lab. Features of patients with primary Sjogren's syndrome.

\begin{tabular}{|c|c|c|c|}
\hline & Age $\square \mathbf{4 0}(\mathbf{n = 6})$ & Age $>$ 40 (n=9) & p value \\
\hline Dry eye & $4(66.7 \%)$ & $7(77.8 \%)$ & $\mathrm{ns}$ \\
\hline Dry mouth & $4(66.7 \%)$ & $7(77.8 \%)$ & $\mathrm{ns}$ \\
\hline Articular involvement & $4(66.7 \%)$ & $6(66.7 \%)$ & $\mathrm{ns}$ \\
\hline Peripheral neuropathy & $1(16.7 \%)$ & $3(33.3 \%)$ & $\mathrm{ns}$ \\
\hline Salivary Gland Biopsy & $4(66.7 \%)$ & $7(77.8 \%)$ & $\mathrm{ns}$ \\
\hline Peripheral Neuropathy & $1(16.7 \%)$ & $3(33.3 \%)$ & $\mathrm{ns}$ \\
\hline Rheumatoid factor & $5(83.3 \%)$ & $3(33.3 \%)$ & $\mathrm{ns}$ \\
\hline Anti SS-A antibody & $5(83.3 \%)$ & $4(44.4 \%)$ & $\mathrm{ns}$ \\
\hline Anti SS-B antibody & $5(83.3 \%)$ & $4(44.4 \%)$ & $\mathrm{ns}$ \\
\hline
\end{tabular}

